\title{
Abdominoperineal Resection with Absorbable Mesh Repair of Perineal Defect for Fournier's Gangrene: A Case Report
}

This article was published in the following Dove Press journal: International Medical Case Reports Journal

\author{
James Holden ${ }^{1,2}$ \\ Jasmir G Nayak ${ }^{1,2}$ \\ Colin Botkin ${ }^{1,2}$ \\ Ramzi M Helewa ${ }^{1,2}$ \\ 'Department of Surgery, University of \\ Manitoba, Winnipeg, Manitoba, Canada; \\ ${ }^{2}$ St. Boniface Hospital, Winnipeg, \\ Manitoba, Canada
}

Background: Fournier's gangrene (FG) is a rare but deadly form of necrotizing fasciitis involving the genital, perineal, and anorectal region. Risk factors include diabetes mellitus, immunosuppression, and alcohol misuse. Because multisystem organ failure can rapidly develop, early diagnosis is critical. Treatment includes fluid resuscitation, broad-spectrum antibiotics, and surgical debridement. Uncommonly, extension of perineal infection into adjacent organs can necessitate multivisceral resection, which can make reconstruction a challenge. Even with swift diagnosis and optimal treatment, morbidity and mortality are high.

Case Presentation: A 66-year-old male with a history of diabetes mellitus presented to the emergency department with progressive scrotal pain, swelling, and perineal skin changes Examination revealed necrosis of the scrotal soft tissues with involvement of the anal canal and rectum. The patient was initiated on intravenous fluids and broad-spectrum antibiotics, then brought immediately to the operating room where surgical care was provided by a urologist, colorectal surgeon, and general surgeon with expertise in complex mesh repair. Extension of necrotic changes travelling proximally through the full thickness of the rectum was noted. The patient underwent extensive scrotal and perineal debridement, laparotomy, abdominoperineal resection (APR), end colostomy, and polyglactin mesh repair of the resultant pelvic floor defect. The patient had appropriate return of bowel function and satisfactory healing of the perineum postoperatively but ultimately died after a ventricular fibrillation-related cardiac arrest precipitated by a flare of idiopathic pulmonary fibrosis.

Conclusion: Early diagnosis and referral to the appropriate specialists are essential elements of managing FG. Here we present a case with extension of necrotizing soft tissue infection into the rectum, requiring pelvic dissection and APR as well as absorbable mesh use to aid in perineal closure. Despite expedient treatment, poor outcomes with this condition are unfortunately common.

Keywords: necrotizing fasciitis, perineal closure, absorbable mesh

\section{Introduction}

Fournier's gangrene was originally named after French dermatologist Jean-Alfred Fournier who, in 1883 , described a case series of previously healthy young males with severe, rapid-onset, necrotizing genital and perineal infections. ${ }^{1}$ FG is most often a polymicrobial process, including a mixture of both aerobic and anaerobic organisms such as Escherichia, Klebsiella, Staphylococcus, Streptococcus, Clostridium, and Bacteroides. ${ }^{2}$ Necrotizing infection most commonly arises from
Correspondence: Ramzi M Helewa Email rhelewa@sbgh.mb.ca
International Medical Case Reports Journal 2021:14 I33-138 
the skin, genitourinary system, or gastrointestinal tract, with bacterial exotoxins and enzymes causing small vessel thrombosis and dermal necrosis. FG shows a significant predilection for males, and its other risk factors include diabetes mellitus, alcohol misuse, chemotherapy, liver disease, and chronic steroid use. ${ }^{3}$ The infection may travel along fascial planes, and involvement of the abdominal wall or visceral organs is an infrequent but recognized finding. ${ }^{4}$ Therapy involves aggressive intravenous fluid resuscitation, broad-spectrum antibiotics, and early surgical debridement of any devitalized tissue. In spite of improvements in the recognition of this condition as well as advances in both critical care and surgical care, mortality remains high (up to $30 \%$ in some studies). ${ }^{5}$

Here we describe a case of FG in a male patient with Type 2 diabetes mellitus who presented to the emergency department with 4 days of progressive scrotal pain. Extension of the necrotizing infection into the anal canal and rectum prompted multidisciplinary involvement during debridement and subsequent surgical reconstruction.

\section{Case Presentation}

A 66-year-old man presented to the emergency room with four days of rectal pain and swelling, culminating in several hours of extreme scrotal pain with an inability to sit down or place any weight on the perineal region. The patient had been generally well prior to these symptoms with no identifiable triggering event. He had been having some difficulty with urination, endorsing low overall urine output and frequent small-volume voids for several days. He had been passing dark bowel movements and reported a small amount of hematochezia prior to hospital presentation. The patient had been taking a topical preparation for a diagnosis of hemorrhoidal bleeding. He did endorse constipation symptoms ongoing for several months, for which he had been taking laxative medications. In fact, the patient had undergone an outpatient esophagogastroduodenoscopy on the morning of his emergency room presentation for the workup of intermittent upper abdominal pain and cramping.

The patient's past medical history included gastroesophageal reflux disease, hypertension, Type 2 diabetes mellitus, coronary artery disease (with a myocardial infarction and stenting 15 years prior), and idiopathic pulmonary fibrosis (IPF). He was known to be colonized with methicillin-resistant Staphylococcus aureus (MRSA). He had no previous abdominal or anorectal surgical history. He took prescription medications including metoprolol, metformin, amitriptyline, omeprazole, glyburide, ramipril, fluoxetine, and insulin. He had an allergy to penicillin resulting in a rash. He did not smoke.

On presentation to the emergency department, the patient's vital signs demonstrated tachycardia (121 beats per minute) and tachypnea (25 respirations per minute), with other vital signs falling within normal limits on room air. Based on the suspicion for Fournier's Gangrene, the patient received intravenous fluids, broad-spectrum antibiotics (piperacillin-tazobactam, clindamycin, and vancomycin), and urology consultation. At the time of urology assessment, all vital signs were all within normal limits on room air. He was awake, alert, and oriented with no signs of distress at rest. The scrotum appeared black and necrotic with extension of gangrenous changes down to the perineum and into the anal canal (Figure 1). There was significant tenderness to any palpation throughout the scrotum and perineum. The abdomen was soft, nondistended, and non-peritonitic.

Bloodwork demonstrated significant leukocytosis with a white blood cell count of $31.3 \times 10^{9}$ cells/L. Imaging was not performed, and the decision was made to bring the patient emergently to the operating room by urology based on clinical examination.

The patient was brought emergently to the operating room. He underwent an extensive scrotal and perineal debridement by the urology team. General/colorectal surgeon consultation was obtained intraoperatively due to extension of necrotic disease into the anus and rectum. The patient underwent further perineal debridement by the general surgery team (Figure 2). Given the presence of necrosis travelling into the ischioanal fossa and along the rectum, the decision was made to proceed with an abdominoperineal resection through a midline laparotomy approach. Surgical specimens included scrotal tissue, sigmoid colon/rectum/anus, and pelvic floor musculature. Because of the degree of levator ani musculature and ischioanal tissue resected, a sizeable defect remained that was not amenable to primary closure. At this point, the expertise of a general surgeon who specializes in complex abdominal wall reconstruction was sought for perineal closure because of the large gap in the pelvic floor left after resection of all devitalized tissue. Placement of a Vicryl (Ethicon Inc., Somerville, New Jersey, United States) mesh was used to aid in reconstruction of the pelvic floor. This was positioned with an inlay technique against the levator ani muscles, adequately providing tissue coverage and preventing organ evisceration. The 


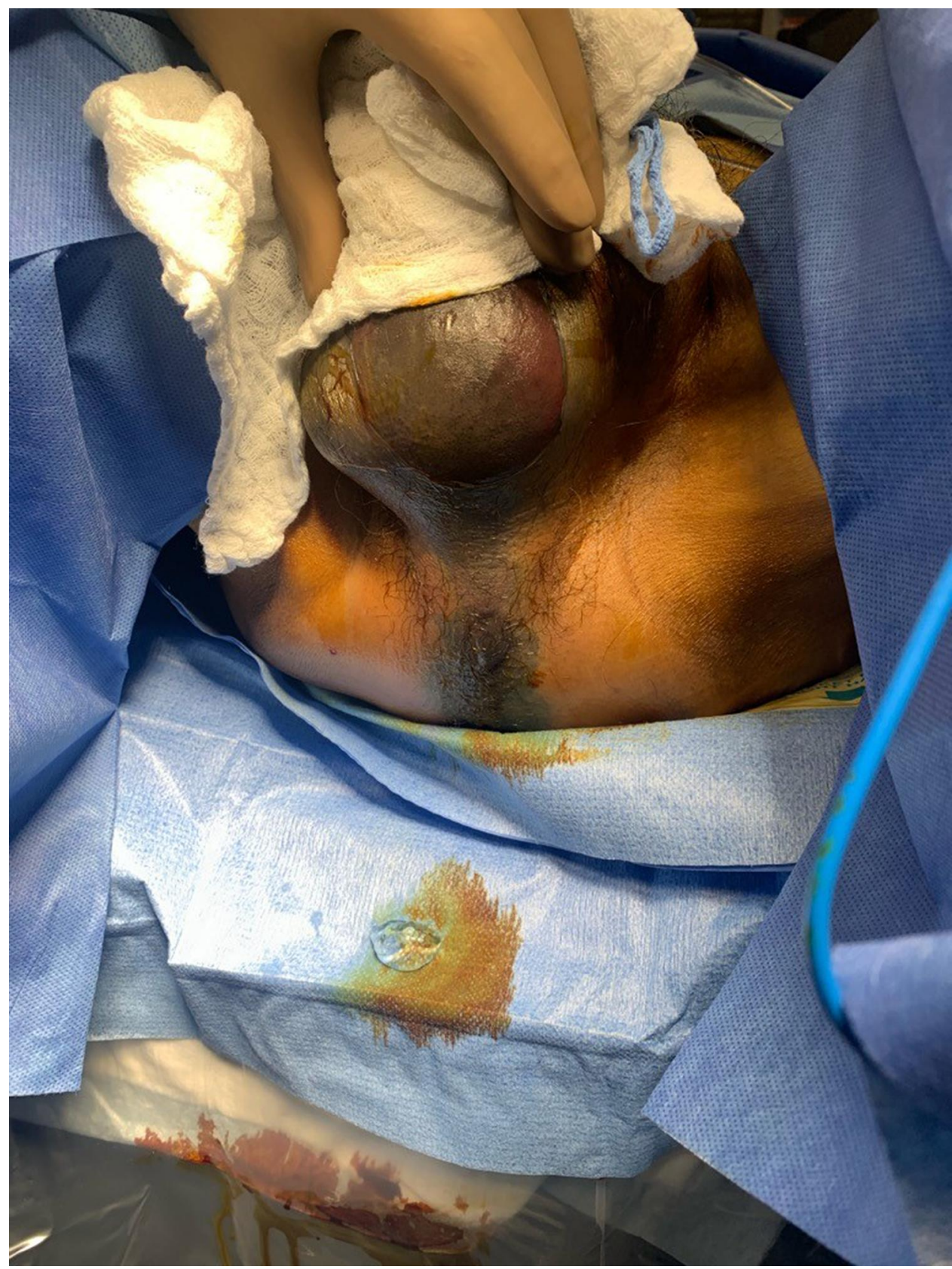

Figure I Necrotizing perineal infection extending from the scrotum into the anal canal.

superficial perineal soft tissue was packed with the skin edges left open to allow healing by secondary intention.

The patient remained on broad-spectrum intravenous antibiotics postoperatively with input from an infectious diseases specialist. He required bedside perineal debridement on postoperative day 7 but otherwise had appropriate wound healing without evidence of dehiscence, hernia, or recurrent necrotizing infection. His postoperative course was complicated by oliguric acute kidney injury and IPF flare-related hypoxia. The patient developed ventricular fibrillation and had a witnessed cardiac arrest on postoperative day 14. Return of spontaneous circulation was achieved but the patient did not have significant neurologic function thereafter. The patient was treated with comfort measures beginning on postoperative day 21 and he passed away peacefully the following day in the presence of family. The perineum was healing well during the initial postoperative period, but we cannot comment on long-term healing results. Final surgical pathology demonstrated soft tissue inflammation and 


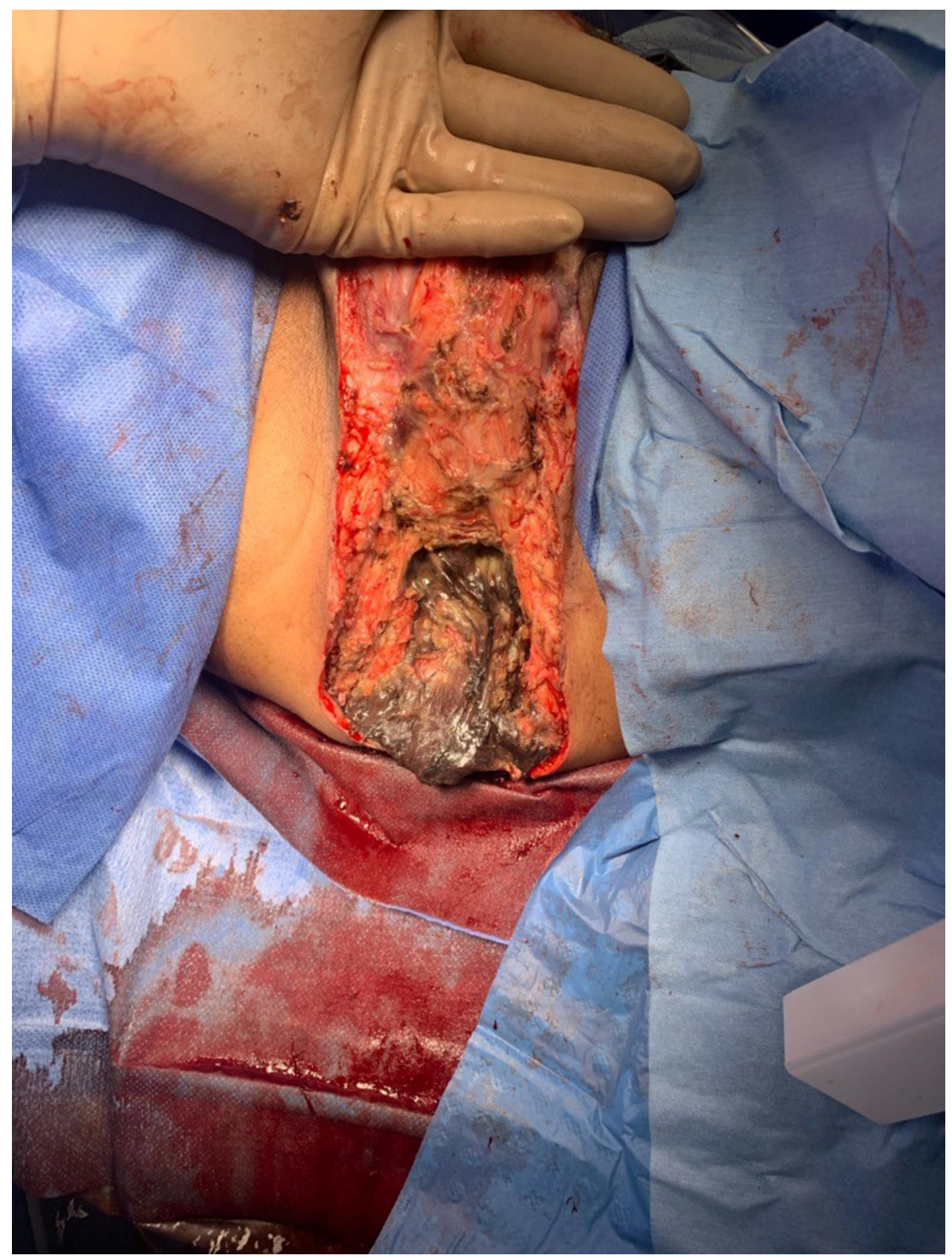

Figure 2 Patient following scrotal and perineal debridement.

necrosis with bacterial colonies, consistent with necrotizing fasciitis. Necrotizing changes travelled from the perineum to the mesorectum, with a full-thickness posterior perforation in the distal rectum.

\section{Discussion}

FG is a form of necrotizing fasciitis affecting the perineum and genitals and is associated with high morbidity and mortality. It may originate from infections that are urogenital, gastrointestinal, or dermatologic/traumatic, though a small proportion of cases are idiopathic. ${ }^{6}$ The disease is more common in men, and one American study estimated an incidence of 1.6 cases per 100000 males per year. ${ }^{7}$ Diabetes mellitus is a key risk factor.

APR has rarely been described as a treatment for FG that develops after hemorrhoidectomy, or in relation to rectal cancer perforation among patients receiving neoadjuvant treatments such as bevacizumab. ${ }^{8,9}$ To the knowledge of these authors, this represents the first literature description of APR required for FG of idiopathic etiology. Regardless of 
the initial source of perineal infection, extension of necrotic changes into the anus and rectum necessitates resection of these organs for adequate disease treatment.

After elective APR, depending on patient and disease factors as well as the pelvic floor defect size, several techniques for perineal closure have been described. These include primary closure, omentoplasty, musculocutaneous flap, and various mesh techniques. ${ }^{10}$ Given the significant soft tissue defect and infected surgical field present in the above case, absorbable mesh repair provided an excellent means by which to reconstitute the pelvic floor without risking flap failure or chronic infection of a synthetic mesh.

A short case series described laparoscopically-placed biologic mesh repair for three patients with simultaneous incarcerated inguinal hernias and findings of FG. ${ }^{11}$ Absorbable mesh repair and mesh placement in a perineal location after surgical treatment of $\mathrm{FG}$ have not been described in the literature until now. Certainly, the use of permanent synthetic mesh materials is best avoided in an infected surgical field, but an absorbable mesh technique represents a feasible option for perineal closure. Absorbable synthetics such as polyglactin are suitable materials for emergency repair when a large defect in a contaminated field requires closure. ${ }^{12}$ One previous study examined the feasibility of polyglactin mesh repair for large abdominal wall defects for patients with enterocutaneous fistula in the setting of an open abdomen, demonstrating satisfactory short-term results. ${ }^{13}$ Although the patient presented here ultimately suffered a mortality related to a postoperative respiratory complication, this case represents a proof-ofconcept of the utility of absorbable mesh for perineal closure in FG. Larger series would be needed to further evaluate the outcomes and durability of this reconstruction option.

\section{Conclusion}

FG is a rare but serious necrotizing infection of the genital, perineal, and anorectal region associated with significant morbidity and mortality. Early recognition and involvement of relevant surgical and critical care specialists is key. Operative debridement is a mainstay of treatment, and a careful evaluation of the extent of necrotic changes must be performed, because involvement of the anus and rectum can rarely necessitate APR. Reconstruction options in an infected field are limited but absorbable polyglactin mesh repair is one viable choice to facilitate pelvic floor closure in such cases.

\section{Abbreviations}

FG, Fournier's gangrene; APR, abdominoperineal resection; IPF, idiopathic pulmonary fibrosis; MRSA, methicillin-resistant Staphylococcus aureus.

\section{Ethics Approval and Informed Consent for Publication}

Informed consent for publication of the patient's medical details and photographs was obtained from the patient's next of kin. Institutional approval was obtained to publish this case report from the University of Manitoba's Health Research Ethics Board.

\section{Author Contributions}

All authors made a significant contribution to the work reported, whether that is in the conception, study design, execution, acquisition of data, analysis and interpretation, or in all these areas; took part in drafting, revising or critically reviewing the article; gave final approval of the version to be published; have agreed on the journal to which the article has been submitted; and agree to be accountable for all aspects of the work.

\section{Funding}

There was no funding for this work.

\section{Disclosure}

The authors declare that they have no conflicts of interest for this work.

\section{References}

1. Mallikarjuna MN, Vijayakumar A, Patil VS, Shivswamy BS Fournier's gangrene: current practices. ISRN Surg. 2012;2012:94 2437. doi: $10.5402 / 2012 / 942437$

2. Yaghan RJ, Al-Jaberi TM, Bani-Hani I. Fournier's gangrene. Dis Colon Rectum. 2000;43(9):1300-1308. doi:10.1007/BF02237442

3. Thwaini A, Khan A, Malik A, et al. Fournier's gangrene and its emergency management. Postgrad Med J. 2006;82(970):516-519. doi:10.1136/pgmj.2005.042069

4. Jeong HJ, Park SC, Seo IY, Rim JS. Prognostic factors in Fournier gangrene. Int J Urol. 2005;12(12):1041-1044. doi:10.1111/j.1442-20 42.2005.01204.x

5. Pawłowski W, Wroński M, Krasnodebski IW. Fournier's gangrene. Pol Merkur Lekarski. 2004;17(97):85-87.

6. Eke N. Fournier's gangrene: a review of 1726 cases: fournier's gangrene. Br J Surg. 2000;87(6):718-728. doi:10.1046/j.1365-2168.2000. 01497.x 
7. Sorensen MD, Krieger JN, Rivara FP, et al. Fournier's Gangrene: population based epidemiology and outcomes. J Urol. 2009;181 (5):2120-2126. doi:10.1016/j.juro.2009.01.034

8. Lehnhardt M, Steinstraesser L, Druecke D, Muehlberger T, Steinau HU, Homann HH. Fournier's gangrene after MilliganMorgan hemorrhoidectomy requiring subsequent abdominoperineal resection of the rectum: report of a case. Dis Colon Rectum. 2004;47 (10):1729-1733. doi:10.1007/s10350-004-0616-x

9. Kobayashi D, Masubuchi M, Takase T, Ichikawa T, Deguchi T, Yaguchi T. Fournier's gangrene caused by penetration of a rectal cancer followed by neoadjuvant chemotherapy. Surg Case Rep. 2018;4(1):123. doi:10.1186/s40792-018-0526-0

10. Peirce C, Martin S. Management of the perineal defect after abdominoperineal excision. Clin Colon Rectal Surg. 2016;29(2):160-167. doi:10.1055/s-0036-1580627
11. Guzzo JL, Bochicchio GV, Henry S, Keller E, Scalea TM. Incarcerated inguinal hernia in the presence of Fournier's gangrene: a novel approach to a complex problem. Am Surg. 2007;73(1):93-95. doi:10.1177/000313480707300122

12. Poussier $\mathrm{M}$, Denève $\mathrm{E}$, Blanc $\mathrm{P}$, et al. A review of available prosthetic material for abdominal wall repair. $J$ Visc Surg. 2013;150(1):52-59. doi:10.1016/j.jviscsurg.2012.10.002

13. Connolly PT, Teubner A, Lees NP, et al. Outcome of reconstructive surgery for intestinal fistula in the open abdomen. Ann Surg. 2008;247(3):440-444. doi:10.1097/SLA.0b013e3181612c99

\section{Publish your work in this journal}

The International Medical Case Reports Journal is an international, peer-reviewed open-access journal publishing original case reports from all medical specialties. Previously unpublished medical posters are also accepted relating to any area of clinical or preclinical science. Submissions should not normally exceed 2,000 words or 4 published pages including figures, diagrams and references. The manuscript management system is completely online and includes a very quick and fair peer-review system, which is all easy to use. Visit http://www.dovepress.com/testimonials.php to read real quotes from published authors. 